\begin{tabular}{|c|c|c|}
\hline & Int.J.Curr.Microbiol.App.Sci (2017) 6(6): 3027-3035 & \\
\hline EXCELLENT & $\begin{array}{l}\text { International Journal of Current Microbiology and Applied Sciences } \\
\text { ISSN: 2319-7706 Volume } 6 \text { Number } 6 \text { (2017) pp. 3027-3035 } \\
\text { Journal homepage: } \underline{\text { http://www.ijcmas.com }}\end{array}$ & 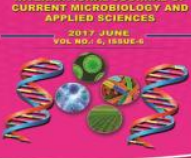 \\
\hline $\begin{array}{l}\text { EXCELLENT } \\
\text { PUBLISHERS }\end{array}$ & & \\
\hline
\end{tabular}

Original Research Article

https://doi.org/10.20546/ijcmas.2017.606.360

\title{
Genetic Variability, Correlation and Path Analysis in Pumpkin (Cucurbita moschata Duch. ex. Poir)
}

\author{
S. Sampath ${ }^{1}$ and V. Krishnamoorthy ${ }^{2 *}$ \\ ${ }^{1}$ Department of Horticulture, AC\&RI, TNAU, Madurai-625 104, India \\ ${ }^{2}$ Department of Vegetable Crops, HC\&RI, TNAU, Coimbatore-641 003, India \\ *Corresponding author email id:
}

\begin{abstract}
A B S T R A C T
The present investigation was carried out at Department of Horticulture, Agricultural College and Research Institute, Tamil Nadu Agricultural University, Madurai during kharif 2015 with 32 pumpkin (Cucurbita moschata Duch. ex. Poir) collected from Keywords

Cucurbita different parts of Tamil Nadu. The genotypes were evaluated for variability estimates, heritability, genetic advance as percent of mean, magnitude of association between characters, their inter-dependence, direct and indirect effects of yield attributes on yield

moschata, Pumpkin, Genotypic and phenotypic coefficient. per plant. High estimates of genotypic and phenotypic coefficient of variation were observed for yield per plant, vine length, number of fruits per plant, fruit weight and 100 seed weight. High heritability with high genetic advance was recorded for number of primary branches, vine length, petiole length, inter node length, first male flower node, sex ratio, number of fruits per plant, fruit length, fruit diameter, flesh thickness, fruit weight, 100 seed weight and yield per plant. The correlation analysis of growth parameters showed that leaf length and days to first female flowering recorded positive significant association with fruit yield. The yield traits revealed that, the traits days to fruit maturity, fruit length, fruit diameter, fruit weight and 100 seed weight showed high positive significant association with fruit yield per plant. Path analysis revealed that vine length, leaf length, inter node length, first male flower node, days to first male flowering, days to first female flowering, sex ratio, days to fruit maturity, number of fruits per plant, fruit length, fruit weight registered positive direct effect on yield.
\end{abstract}

\section{Introduction}

Pumpkin is one of the most important cucurbitaceous vegetable crop grown throughout India under a wide range of agro climatic conditions and is known for its high carotene content in the fruit. In our country, a wide range of variability for vegetative and fruit character is available in pumpkin. The monoecious character, conspicuous and solitary flowers, large seed number of seed per fruit and wide variability for yield, size and shape of fruit prompted the breeder to exploit this crop commercially. Being a crosspollinated crop, pumpkin has a wide range of variability for maturity, yield and fruit characters like shape and size. During the last two decades many workers utilized breeding as a tool for improvement of yield in pumpkin (Sirohi and Ghorui, 1993). However, the genetic potential of this crop needs further exploitation to its nearest perfection. Studies on the genetic variability for yield and its component characters are the pre requisite for 
a successful crop improvement programme. Phenotypic variability being highly influenced by the environment, does not give a real picture of the potential genotypic variability. Hence, knowledge about heritability and genetic advance on yield and yield contributing characters are necessary for the crop improvement through selection. Further, yield is a complex quantitative trait governed by large number of genes and is also greatly influenced by environmental factors. Hence, selection of superior accessions based on yield as such is not effective. Therefore, association analysis between different traits and path analysis, which partitions the correlation into direct and indirect effects, should be studied. Therefore, an attempt was made to study the variance, heritability and genetic advance of different characters. The association analysis and path coefficient analysis were also carried out to know the contribution of all the characters towards fruit yield.

\section{Materials and Methods}

The study was conducted at Department of Horticulture, Agricultural College and Research Institute, Tamil Nadu Agricultural University, Madurai, Tamil Nadu, India during kharif 2015. The details of the genotypes used for the study were CM2, CM3, CM5, CM6, CM7, CM8, CM9, CM10, CM11, CM12, CM13, CM14, CM15, CM17, CM18, CM20, CM21 were maintained in the department of horticulture, the genotypes collected from where CM1 is Attur, Salem district, CM4 is Melur, Madurai district, CM16 is Olakkur, Villupuram district, CM19 is Gudalur, Theni district, $\mathrm{CM} 22$ is Gudiyatham, Vellore district, CM23 is Thirumangalam, Madurai district, CM24 id Natham, Dindugal district, CM25 is Vikravandi, Villupuram District,CM26 is Arani, Thiruvanamalai district, CM27 Sempatti, Dindugal district, CM28 is
Ottamchatram, Dindugal district, CM29 is Rajapalayam, Virdhunagar district,CM30 is Tholudur, Perambalur District, CM31 is Harur, Dharmapuri district and CM 32 is Co1 variety. The seeds were sown in pits taken at a row spacing of $2.0 \mathrm{mt}$ and intra row spacing of $2.0 \mathrm{~m}$ in randomized block design. The observations were recorded in five randomly selected plants from each replication for the genotypes were evaluated for following characters viz., number of primary branches, vine length $(\mathrm{cm})$, petiole length $(\mathrm{cm})$, leaf length $(\mathrm{cm})$, leaf breadth $(\mathrm{cm})$, inter node length $(\mathrm{cm})$, first male flower node, first female flower node, days to first male flowering, days to first female flowering, sex ratio, days to fruit maturity, number of fruits per plant, fruit length $(\mathrm{cm})$, fruit diameter $(\mathrm{cm})$, flesh thickness $(\mathrm{cm})$, fruit weight $(\mathrm{g})$, 100 seed weight $(\mathrm{g})$, yield per plant $(\mathrm{kg})$. The data recorded were subjected to genotypic coefficient of variation (GCV), phenotypic coefficient of variation (PCV), broad sense heritability, correlation (genotypic and phenotypic) and path coefficient were computed by the methods suggested by Panse and Sukhatme (1967).

\section{Results and Discussion}

\section{Variability}

Relatively high estimates of genotypic and phenotypic coefficient of variation were observed for yield per plant $(33.88 \%)$, vine length $(27.50 \%)$, number of fruits per plant (29.16\%), fruit weight $(44.21 \%)$ and 100 seed weight $(23.20 \%)$. High phenotypic coefficient of variation was recorded for vine length $(31.36 \%)$, sex ratio, fruit weight $(44.69 \%)$, yield per plant $(40.27 \%)$, number of fruits per plant $(39.85 \%)$ and 100 seed weight $(23.38 \%)$. This is in accordance with the findings Samadia (2011) in ridge gourd for fruit length and number of fruits per vine. For fruit girth, similar results were reported by 
Ahmed et al., (2005) in bottle gourd, Dey et al., (2009) in bitter gourd observed similar results for average fruit weight.

Moderate genotypic coefficients of variation was observed for number of primary branches (15.18\%), petiole length $(11.10 \%)$, inter node length $(15.86 \%)$, first male flower node $(15.13 \%)$, sex ratio (19.84\%), fruit length $(17.0 \%)$ and flesh thickness (12.18\%). Moderate phenotypic coefficient of variation was observed for number of primary branches (16.98\%, petiole length $(12.07 \%)$, leaf length $(10.47 \%)$, leaf breadth $(11.32 \%)$, first male flower node $(17.62 \%)$, inter node length $(17.79 \%)$, sex ratio $(20.01 \%)$, fruit length $(17.79 \%)$, fruit diameter $(11.08 \%)$ and flesh thickness $(13.77 \%)$.. This is in corroboration with the findings of Punitha (2000) in cucumber for vine length and Sahithya (2001) in snake gourd.

Low estimates of genotypic coefficient of variation was observed for leaf length $(8.73 \%)$, leaf breadth $(8.47 \%)$, first male flower node $(15.13 \%)$, days to first male flowering $(2.25 \%)$, days to first female flowering (3.20) and days to fruit maturity $(1.44 \%)$ and low phenotypic coefficient of variation for first female flower node $(8.51 \%)$, days to first male flowering $(5.54 \%)$, days to first female flowering $(4.25 \%)$ and days to fruit maturity $(3.08 \%)$. Similar results were reported by Kumar et al., (2007) in bottle gourd for days to first female flower anthesis and days to first male flower anthesis.

\section{Heritability and genetic advance}

The high heritability coupled with high genetic advance was observed for primary branches (79.93 and $27.96 \%$ ), vine length (76.87 and $49.66 \%)$, petiole length (84.54 and $21.03 \%)$, inter node length (79.52 and $29.14 \%$ ), first male flower node (73.72 and $26.76 \%$ ), sex ratio (98.25 and $40.50 \%$ ), number of fruits per plant (70 and $50.27 \%$ ), fruit length (91 and $33.40 \%$ ), fruit diameter (94.90 and $21.66 \%$ ), flesh thickness (78.20 and $22.10 \%)$, fruit weight $(97.80$ and $90.09 \%), \quad 100$ seed weight $(98.50$ and $47.44 \%$ ) and yield per plant (71.00 and $58.82 \%)$. This result is in conformity with the findings of Dhatt and Singh (2008) in pumpkin. Further high heritability coupled with high expected genetic advance indicated the involvement of additive genetic variance, selection may be effective for improvement of these traits. Similar findings were reported by Kumar et al., (2010) (Tables 1-3).

\section{Correlation studies}

\section{Correlation studies between fruit yield and its component traits}

The genotypic correlation coefficient worked out among different vegetative characters revealed that, out of twelve characters studied, only leaf length (0.335) and days to first female flowering (0.414) recorded positive significant association with fruit yield. Similar trend of correlation was reported by Rakhi and Rajamony (2005) in muskmelon. With respect to yield related traits days to fruit maturity (0.339), fruit length (0.524), fruit diameter (0.511), fruit weight (0.817) and 100 seed weight (0.175) showed high positive significant association with fruit yield. While exercising selection, emphasis must be laid on vine length, leaf length, days to first female flowering, sex ratio, days to fruit maturity, fruit diameter, fruit weight and 100 seed weight. These characters provide simultaneous improvement on fruit yield.

\section{Inter correlations among important yield attributing components}

The inter correlation among vegetative characters revealed significant and positive value for leaf length with number of primary 
branches $(0.564 \%)$ and leaf breath, days to first female flowering with number of branches per plant (0.309) and vein length (0.619). Yield traits revealed significant and positive value for fruit length with days to fruit maturity (0.299) whereas, Fruit diameter days to maturity (0.665) and fruit length (0.681) yield per plant. With respect to fruit weight, it had positive correlation with 100 seed weight (0.339) and yield per plant
(0.817). Also days to fruit maturity had significant had positive correlation with fruit length (0.299), fruit diameter (0.665), flesh thickness (0.856), fruit weight (0.298) and yield per plant (0.339), whereas, fruit weight had positive correlation with 100 seed weight (0.428) and yield per plant (0.339). These results are in corroboration with Singh and Rajeshkumar (2002) in bottle gourd (Tables 3 and 4).

Table.1 Phenotypic and genotypic coefficient variability, heritability, genetic advance as per cent of mean of pumpkin genotypes for vegetative traits

\begin{tabular}{|l|c|c|c|c|}
\hline \multicolumn{1}{|c|}{ Characters } & $\begin{array}{c}\text { Genotypic coefficient } \\
\text { variation } \\
\text { (GCV \%) }\end{array}$ & $\begin{array}{c}\text { Phenotypic } \\
\text { coefficient } \\
\text { variation (PCV } \\
\text { \%) }\end{array}$ & $\begin{array}{c}\text { Heritabil } \\
\text { ity } \\
(\%)\end{array}$ & $\begin{array}{c}\text { Percentage of } \\
\text { contribution }\end{array}$ \\
\hline $\begin{array}{l}\text { Number of primary } \\
\text { branches per plant }\end{array}$ & 15.18 & 16.98 & 79.93 & 27.96 \\
\hline Vine length (cm) & 27.50 & 31.36 & 76.87 & 49.66 \\
\hline Petiole length (cm) & 11.10 & 12.07 & 84.54 & 21.03 \\
\hline Leaf length (cm) & 8.73 & 10.47 & 69.63 & 15.01 \\
\hline Leaf breadth (cm) & 8.47 & 11.32 & 55.97 & 13.05 \\
\hline Inter node length (cm) & 15.86 & 17.79 & 79.52 & 29.14 \\
\hline First male flower node & 15.13 & 17.62 & 73.72 & 26.76 \\
\hline First female flower node & 7.10 & 8.51 & 69.69 & 12.22 \\
\hline Days to first male flowering & 2.25 & 5.54 & 16.55 & 1.89 \\
\hline $\begin{array}{l}\text { Days to first female } \\
\text { flowering }\end{array}$ & 3.20 & 4.25 & 56.73 & 4.96 \\
\hline Sex ratio & 19.84 & 20.01 & 98.25 & 40.50 \\
\hline Yield per plant (kg) & 33.88 & 40.27 & 71.04 & 58.82 \\
\hline
\end{tabular}

Table.2 Phenotypic and genotypic coefficient variability, heritability, genetic advance as per cent of mean of pumpkin genotypes for yield and its related attributes

\begin{tabular}{|l|c|c|c|c|}
\hline \multicolumn{1}{|c|}{ Characters } & $\begin{array}{c}\text { Genotypic } \\
\text { coefficient } \\
\text { variation } \\
\text { (GCV \%) }\end{array}$ & $\begin{array}{c}\text { Phenotypic } \\
\text { coefficient } \\
\text { variation } \\
\text { (PCV \%) }\end{array}$ & $\begin{array}{c}\text { Heritability } \\
(\boldsymbol{\%})\end{array}$ & $\begin{array}{c}\text { Percentage of } \\
\text { contribution }\end{array}$ \\
\hline Days to fruit maturity & 1.44 & 3.08 & 22.00 & 1.40 \\
\hline Number of fruits of pr plant & 29.16 & 34.85 & 70.00 & 50.27 \\
\hline Fruit length (cm) & 17.00 & 17.82 & 91.00 & 33.42 \\
\hline Fruit diameter (cm) & 10.79 & 11.08 & 94.90 & 21.66 \\
\hline Flesh thickness (cm) & 12.18 & 13.77 & 78.20 & 22.19 \\
\hline Fruit weight $\mathbf{( k g )}$ & 44.21 & 44.69 & 97.80 & 90.09 \\
\hline 100 seed weight $\mathbf{( g )}$ & 23.20 & 23.38 & 98.50 & 47.44 \\
\hline Yield per plant $\mathbf{( k g )}$ & 33.88 & 40.20 & 71.00 & 58.82 \\
\hline
\end{tabular}


Table.3 Genotypic $(\mathrm{G})$ and phenotypic $(\mathrm{P})$ correlation coefficients in pumpkin genotypes for vegetative traits

\begin{tabular}{|c|c|c|c|c|c|c|c|c|c|c|c|c|c|}
\hline Characters & & $\begin{array}{l}\text { Number } \\
\text { of } \\
\text { primary } \\
\text { branches } \\
\text { per plant }\end{array}$ & $\begin{array}{c}\text { Vine } \\
\text { length } \\
(\mathrm{cm})\end{array}$ & $\begin{array}{c}\text { Petiole } \\
\text { length } \\
(\mathrm{cm})\end{array}$ & $\begin{array}{c}\text { Leaf } \\
\text { length } \\
(\mathrm{cm})\end{array}$ & $\begin{array}{c}\text { Leaf } \\
\text { breadth } \\
(\mathrm{cm})\end{array}$ & $\begin{array}{c}\text { Inter } \\
\text { node } \\
\text { length } \\
(\mathrm{cm})\end{array}$ & $\begin{array}{c}\text { First } \\
\text { male } \\
\text { flower } \\
\text { node }\end{array}$ & $\begin{array}{c}\text { First } \\
\text { female } \\
\text { flower } \\
\text { node }\end{array}$ & $\begin{array}{c}\text { Days to } \\
\text { first male } \\
\text { flower } \\
\text { flowering }\end{array}$ & $\begin{array}{c}\text { Days to } \\
\text { first } \\
\text { female } \\
\text { flowering }\end{array}$ & $\begin{array}{c}\text { Sex } \\
\text { ratio }\end{array}$ & $\begin{array}{c}\text { Yield } \\
\text { per } \\
\text { plant } \\
(\mathbf{k g})\end{array}$ \\
\hline \multirow{2}{*}{$\begin{array}{l}\text { Number of primary } \\
\text { branches per plant }\end{array}$} & $\mathrm{G}$ & 1.000 & -0.035 & 0.015 & $0.564 * *$ & 0.164 & -0.100 & 0.188 & -0.040 & $-0.452 * *$ & $0.309 *$ & 0.077 & 0.211 \\
\hline & $\mathrm{P}$ & 1.000 & -0.032 & -0.042 & $0.434 * *$ & 0.058 & -0.074 & 0.089 & -0.019 & -0.189 & 0.212 & 0.081 & 0.139 \\
\hline \multirow{2}{*}{ Vine length(cm) } & $\mathrm{G}$ & & 1.000 & 0.278 & -0.004 & 0.270 & $-0.305^{*}$ & -0.063 & $-0.413 * *$ & 0.280 & $0.619 * *$ & 0.165 & 0.282 \\
\hline & $\mathrm{P}$ & & 1.000 & 0.224 & -0.020 & 0.182 & -0.201 & -0.076 & -0.295 & 0.039 & $0.336^{*}$ & 0.140 & 0.185 \\
\hline \multirow{2}{*}{ Petiole length $(\mathrm{cm})$} & $\mathrm{G}$ & & & 1.000 & -0.022 & $0.313^{*}$ & 0.233 & -0.060 & 0.134 & 0.072 & 0.133 & 0.028 & -0.266 \\
\hline & $\mathrm{P}$ & & & 1.000 & 0.005 & $0.342 *$ & 0.187 & 0.024 & 0.151 & 0.057 & 0.108 & 0.014 & -0.200 \\
\hline \multirow{2}{*}{ Leaf length (cm) } & $\mathrm{G}$ & & & & 1.000 & $0.413 * *$ & -0.246 & -0.245 & 0.014 & $-0.406^{*}$ & 0.184 & -0.209 & $0.335^{*}$ \\
\hline & $\mathrm{P}$ & & & & 1.000 & $0.305^{*}$ & -0.156 & -0.156 & 0.006 & -0.077 & 0.198 & -0.163 & 0.266 \\
\hline \multirow{2}{*}{ Leaf breadth (cm) } & G & & & & & 1.000 & 0.203 & -0.155 & $0.544 * *$ & $0.641 *$ & 0.170 & -0.043 & -0.177 \\
\hline & $\mathrm{P}$ & & & & & 1.000 & 0.135 & 0.095 & $0.365^{*}$ & 0.154 & 0.060 & -0.021 & -0.047 \\
\hline \multirow{2}{*}{ Inter node length $(\mathrm{cm})$} & $\mathrm{G}$ & & & & & & 1.000 & 0.043 & $0.361 *$ & -0.011 & $-0.470 * *$ & 0.020 & -0.228 \\
\hline & $\mathrm{P}$ & & & & & & 1.000 & -0.015 & 0.294 & 0.056 & $-0.328 *$ & 0.018 & -0.099 \\
\hline \multirow{2}{*}{ First male flower node } & $\mathrm{G}$ & & & & & & & 1.000 & -0.032 & -0.023 & 0.077 & $0.421 * *$ & -0.119 \\
\hline & $\mathrm{P}$ & & & & & & & 1.000 & 0.010 & -0.092 & 0.015 & $0.367 *$ & -0.090 \\
\hline \multirow{2}{*}{ First female flower node } & $\mathrm{G}$ & & & & & & & & 1.000 & $0.643 * *$ & -0.270 & -0.277 & $-0.385^{*}$ \\
\hline & $\mathrm{P}$ & & & & & & & & 1.000 & 0.217 & -0.089 & -0.235 & -0.276 \\
\hline \multirow{2}{*}{$\begin{array}{l}\text { Days to first male } \\
\text { Flowering }\end{array}$} & $\mathrm{G}$ & & & & & & & & & 1.000 & 0.232 & -0.219 & $-0.504 * *$ \\
\hline & $\mathrm{P}$ & & & & & & & & & 1.000 & 0.105 & -0.120 & -0.215 \\
\hline \multirow{2}{*}{$\begin{array}{l}\text { Days to first female } \\
\text { Flowering }\end{array}$} & $\mathrm{G}$ & & & & & & & & & & 1.000 & 0.242 & $0.414 * *$ \\
\hline & $\mathrm{P}$ & & & & & & & & & & 1.000 & 0.171 & 0.257 \\
\hline \multirow{2}{*}{ Sex ratio } & $\mathrm{G}$ & & & & & & & & & & & 1.000 & 0.095 \\
\hline & $\mathrm{P}$ & & & & & & & & & & & 1.000 & 0.084 \\
\hline \multirow{2}{*}{ Yield per plant(kg) } & $\mathrm{G}$ & & & & & & & & & & & & 1.000 \\
\hline & $\mathrm{P}$ & & & & & & & & & & & & 1.000 \\
\hline
\end{tabular}


Table.4 Genotypic (G) and phenotypic (P) correlation coefficients in pumpkin genotypes for yield and its attributes

\begin{tabular}{|c|c|c|c|c|c|c|c|c|c|}
\hline Characters & & $\begin{array}{c}\text { Days to } \\
\text { fruit } \\
\text { maturity }\end{array}$ & $\begin{array}{l}\text { Number of } \\
\text { fruits of per } \\
\text { plant }\end{array}$ & $\begin{array}{l}\text { Fruit length } \\
(\mathrm{cm})\end{array}$ & $\begin{array}{c}\text { Fruit } \\
\text { diameter } \\
(\mathrm{cm})\end{array}$ & $\begin{array}{c}\text { Flesh } \\
\text { thickness } \\
(\mathrm{cm})\end{array}$ & $\begin{array}{c}\text { Fruit } \\
\text { weight } \\
\text { (kg) }\end{array}$ & $\begin{array}{l}100 \text { seed } \\
\text { weight } \\
(\mathrm{g})\end{array}$ & $\begin{array}{c}\text { Yield per } \\
\text { plant } \\
(\mathbf{k g})\end{array}$ \\
\hline \multirow{2}{*}{ Days to fruit maturity } & $\mathrm{G}$ & 1 & $-0.564 * *$ & $0.299 *$ & $0.665 * *$ & $0.856 * *$ & $0.298^{*}$ & $0.428 * *$ & $0.339 *$ \\
\hline & $\mathrm{P}$ & 1 & $-0.297 *$ & 0.160 & 0.310 & 0.390 & 0.091 & 0.201 & 0.044 \\
\hline \multirow{2}{*}{$\begin{array}{l}\text { Number of fruits of per } \\
\text { plant }\end{array}$} & G & & 1 & $-0.559 * *$ & $-0.633 * *$ & $-0.522 * *$ & $-0.496 * *$ & -0.238 & -0.104 \\
\hline & $\mathrm{P}$ & & 1 & $-0.447 *$ & $-0.509 * *$ & $-0.405^{* *}$ & $-0.408 * *$ & -0.196 & 0.190 \\
\hline \multirow{2}{*}{ Fruit length (cm) } & G & & & 1 & $0.681 * *$ & 0.264 & $0.716 * *$ & 0.248 & $0.524 * *$ \\
\hline & $\mathrm{P}$ & & & 1 & $0.642 * *$ & 0.209 & $0.667 * *$ & 0.238 & $0.424 * *$ \\
\hline \multirow{2}{*}{ Fruit diameter (cm) } & $\mathrm{G}$ & & & & 1 & $0.387^{*}$ & $0.687 * *$ & $0.352 *$ & $0.511 * *$ \\
\hline & $\mathrm{P}$ & & & & 1 & $0.341^{*}$ & $0.664 * *$ & $0.345^{*}$ & $0.431 * *$ \\
\hline \multirow{2}{*}{ Flesh thickness (cm) } & G & & & & & 1 & 0.141 & 0.083 & 0.005 \\
\hline & $\mathrm{P}$ & & & & & 1 & 0.122 & 0.078 & -0.024 \\
\hline \multirow{2}{*}{ Fruit weight (kg) } & $\mathrm{G}$ & & & & & & 1 & $0.336^{*}$ & $0.817 * *$ \\
\hline & $\mathrm{P}$ & & & & & & 1 & $0.329 *$ & $0.702 * *$ \\
\hline \multirow{2}{*}{100 seed weight $(g)$} & $\mathrm{G}$ & & & & & & & 1 & 0.175 \\
\hline & $\mathrm{P}$ & & & & & & & 1 & 0.154 \\
\hline \multirow{2}{*}{ Yield per plant (kg) } & $\mathrm{G}$ & & & & & & & & 1 \\
\hline & $\mathrm{P}$ & & & & & & & & 1 \\
\hline
\end{tabular}


Table.5 Path coefficient analysis for direct and indirect effects of pumpkin genotypes for vegetative traits

\begin{tabular}{|c|c|c|c|c|c|c|c|c|c|c|c|c|}
\hline Characters & $\begin{array}{c}\text { Number of } \\
\text { primary } \\
\text { branches } \\
\text { per plant }\end{array}$ & $\begin{array}{c}\text { Vine } \\
\text { length } \\
(\mathrm{cm})\end{array}$ & $\begin{array}{c}\text { Petiole } \\
\text { length } \\
(\mathrm{cm})\end{array}$ & $\begin{array}{c}\text { Leaf } \\
\text { length } \\
(\mathrm{cm})\end{array}$ & $\begin{array}{c}\text { Leaf } \\
\text { breadth } \\
(\mathrm{cm})\end{array}$ & $\begin{array}{l}\text { Inter } \\
\text { node } \\
\text { length } \\
(\mathrm{cm})\end{array}$ & $\begin{array}{c}\text { First } \\
\text { male } \\
\text { flowe } \\
\quad r \\
\text { node }\end{array}$ & $\begin{array}{c}\text { First } \\
\text { femal } \\
e \\
\text { flowe } \\
r \\
\text { node } \\
\end{array}$ & $\begin{array}{c}\text { Days to } \\
\text { first } \\
\text { male } \\
\text { flowerin } \\
\quad \mathrm{g}\end{array}$ & $\begin{array}{c}\text { Days to } \\
\text { first } \\
\text { female } \\
\text { flowerin } \\
\mathrm{g}\end{array}$ & $\begin{array}{l}\text { Sex } \\
\text { ratio }\end{array}$ & $\begin{array}{c}\text { Genotypi } \\
\text { c } \\
\text { correlatio } \\
n \\
\text { coefficien } \\
\text { t yield } \\
\end{array}$ \\
\hline $\begin{array}{l}\text { Number of primary } \\
\text { branches per plant }\end{array}$ & -0.105 & -0.030 & -0.005 & 0.601 & -0.212 & -0.057 & -0.031 & -0.023 & -0.052 & 0.102 & 0.024 & 0.211 \\
\hline Vine length $(\mathrm{cm})$ & 0.004 & 0.851 & -0.099 & -0.005 & -0.350 & -0.175 & 0.010 & -0.242 & 0.032 & 0.204 & 0.050 & 0.282 \\
\hline Petiole length $(\mathrm{cm})$ & -0.002 & 0.237 & -0.355 & -0.023 & -0.405 & 0.133 & 0.010 & 0.079 & 0.008 & 0.044 & 0.009 & -0.266 \\
\hline Leaf length $(\mathrm{cm})$ & -0.059 & -0.004 & 0.008 & 1.066 & -0.534 & -0.141 & 0.040 & 0.008 & -0.047 & 0.061 & -0.064 & 0.335 \\
\hline Leaf breadth $(\mathrm{cm})$ & -0.017 & 0.230 & -0.111 & 0.440 & -1.295 & 0.116 & 0.025 & 0.319 & 0.074 & 0.056 & -0.013 & -0.177 \\
\hline Inter node length $(\mathrm{cm})$ & 0.010 & -0.259 & -0.083 & -0.262 & -0.262 & 0.573 & -0.007 & 0.212 & -0.001 & -0.155 & 0.006 & -0.228 \\
\hline First male flower node & -0.020 & -0.053 & 0.021 & -0.261 & 0.201 & 0.024 & -0.164 & -0.019 & -0.003 & 0.025 & 0.129 & -0.119 \\
\hline $\begin{array}{l}\text { First female flower } \\
\text { node }\end{array}$ & 0.004 & -0.351 & -0.048 & 0.015 & -0.704 & 0.207 & 0.005 & 0.587 & 0.074 & -0.089 & -0.085 & -0.385 \\
\hline $\begin{array}{l}\text { Days to first male } \\
\text { Flowering }\end{array}$ & 0.047 & 0.238 & -0.026 & -0.433 & -0.830 & -0.006 & 0.004 & 0.377 & 0.115 & 0.076 & -0.067 & -0.504 \\
\hline $\begin{array}{l}\text { Days to first female } \\
\text { Flowering }\end{array}$ & -0.032 & 0.527 & -0.047 & 0.196 & -0.220 & -0.269 & -0.013 & -0.158 & 0.027 & 0.329 & 0.074 & 0.414 \\
\hline Sex ratio & -0.008 & 0.141 & -0.010 & -0.223 & 0.055 & 0.012 & -0.069 & -0.163 & -0.025 & 0.080 & 0.305 & 0.095 \\
\hline
\end{tabular}

Residual factor $=0.576658$

Table.6 Path coefficient analysis for direct and indirect effects of pumpkin genotypes for yield and its related attributes

\begin{tabular}{|c|c|c|c|c|c|c|c|c|}
\hline Characters & $\begin{array}{c}\text { Days to } \\
\text { fruit } \\
\text { maturity }\end{array}$ & $\begin{array}{c}\text { Number of } \\
\text { fruits per } \\
\text { plant }\end{array}$ & $\begin{array}{l}\text { Fruit } \\
\text { length } \\
(\mathrm{cm})\end{array}$ & $\begin{array}{c}\text { Fruit } \\
\text { diameter } \\
(\mathrm{cm})\end{array}$ & $\begin{array}{c}\text { Flesh } \\
\text { thickness } \\
(\mathrm{cm})\end{array}$ & $\begin{array}{c}\text { Fruit } \\
\text { weight } \\
\text { (g) }\end{array}$ & $\begin{array}{c}100 \text { seed } \\
\text { weight } \\
(\mathrm{kg}) \\
\end{array}$ & $\begin{array}{c}\text { Genotypic } \\
\text { correlation } \\
\text { coefficient yield }\end{array}$ \\
\hline Days to fruit maturity & 7.447 & -0.091 & 0.572 & -2.506 & -4.578 & 0.407 & -0.912 & 0.339 \\
\hline Number of fruits per plant & -4.201 & 0.162 & -1.072 & 2.384 & 2.792 & -0.678 & 0.508 & -0.105 \\
\hline Fruit length $(\mathrm{cm})$ & 2.225 & -0.090 & 1.916 & -2.566 & -1.411 & 0.979 & -0.529 & 0.524 \\
\hline Fruit diameter $(\mathrm{cm})$ & 4.956 & -0.102 & 1.306 & -3.766 & -2.070 & 0.940 & -0.751 & 0.513 \\
\hline Flesh thickness(cm) & 6.374 & -0.084 & 0.505 & -1.458 & -5.348 & 0.193 & -0.178 & 0.004 \\
\hline Fruit weight (kg) & 2.218 & -0.080 & 1.372 & -2.589 & -0.755 & 1.367 & -0.716 & 0.817 \\
\hline 100 seed weight $(\mathrm{g})$ & 3.184 & -0.038 & 0.475 & -1.325 & -0.445 & 0.459 & -2.134 & 0.176 \\
\hline
\end{tabular}

Residual factor $=0.142831$ 
The conclusion from the association analysis is that, intentional selection based on traits like days to fruit maturity, fruit length, fruit diameter, flesh thickness, fruit weight and 100 seed weight may result in simultaneous improvement of fruit yield per plant and also these traits were inter correlated among themselves. Further, it clearly indicates that these characters are highly reliable components of fruit yield and could very well be utilized as yield indicator while exercising selection.

\section{Path analysis of yield and its component} traits

In the present investigation, the characters viz., vine length (0.851), leaf length (1.066), inter node length $(0.573)$, first female flower node (0.587), days to first male flowering (0.115), days to first female flowering (0.329), sex ratio (0.305), days to fruit maturity (7.447), number of fruits per plant (0.162), fruit length (1.916), fruit weight (1.367), registered positive direct effect on yield. Similar results were observed by Shivananda et al., (2013) in pumpkin, Rao et al., (2000) and Prabha et al., (2008) in ridge gourd for number of seeds per fruit. The direct selections for these characters are likely to bring about an overall improvement in fruit yield per plant (Tables 4 and 5).

In the present study, the characters viz., number of primary branches $(-0.105)$, petiole length (-0.355), leaf breadth $(-1.295)$, first male flower node $(-0.164)$, fruit diameter (3.766), flesh thickness (-5.348), 100 seed weight $(-2.134)$ and ascorbic acid (-0.128) were registered negative direct effect on yield. Similar results were observed by Choudhary et al., (2003) for vine length and days to first male flower anthesis in muskmelon and Yadav et al., (2010) for number of primary branches per plant in bottle gourd.
The result of path analysis study revealed that the characters like fruit weight (1.367) and flesh thickness (-5.348) were the most important yield determinants, because of their high direct effects and high indirect effects via many other yield and quality improving characters. The indirect effect also showed that most of the characters influenced the yield through vine length (0.282), days to first female flower anthesis (0.414) and fruit length (0.524). This suggests that emphasis must be given to such traits while exercising selection to improve the yield in pumpkin.

\section{References}

Ahmed, N., Z. Hakeem, B. Afroza, R. Narayan and Faheema S. 2005. Variability studies in bottle gourd. Haryana J. Hort. Sci., 34 (3-4): 336337.

Choudhary, B.R., R.S. Dhaka and Fageria, M.S. 2003. Correlation and path coefficient analysis in muskmelon. Haryana J. Hort. Sci., 32 (1\&2): 98101.

Dey, S.S., T.K. Behera, A.D. Munshi and Bhatia R. 2009. Genetic variability, genetic advance and heritability in bitter gourd (Momordica charantia L.). Indian Agric., 53 (1\&2): 7-12.

Dhatt, A.S. and Singh, H. 2008. Genetic variability, heritability and path coefficient analysis in pumpkin. Crop Improvement. 35 (1): 91-94.

Kumar, J., A.D. Munshi, R. Kema and Sureja, A.K. 2010. Studies on Heterosis in slicing cucumber. Indian J. Hort., 67 (2): 197-201.

Kumar, S., R. Singh and Pal, A.K. 2007. Genetic variability, heritability, genetic advance, correlation coefficient and path analysis in bottle gourd. Indian J. Hort., 64 (2): 163-168.

Panse, V.G and Sukhatme, P.V. 1967. Statistical methods for agricultural 
workers. ICAR, New Delhi. p. 134-192. Prabha, R.J., T.P. Latha, C.R. Sankar and Rao, V.S. 2008. Character association and path coefficient studies in ridge gourd [Luffa acutangula (Roxb.) L.]. The Andhra Agric. J., 55 (1): 63-67.

Punitha, A. 2000. Genetic variability studies in cucumber (Cucumis sativus L.) M.Sc. (Hort.) Thesis, Tamil Nadu Agricultural University, Madurai.

Rakhi, R and Rajamony, L. 2005. Variability, heritability and genetic advance in landraces of culinary melon (Cucumis melo L.). J. Trop. Agric., 43 (1-2): 79-82.

Rao, N.L., B.P.V. Rao and Reddy, I.P. 2000. Character association and path correlation studies in ridge gourd [Luffa acutangula (Roxb.) L.]. The Andra Agric. 47 (1-2): 103-107.

Sahithya, 2001.Genetic evaluation of $F_{2}$ and $\mathrm{F}_{3}$ generations in snake gourd
(Trichosanthes anguina L.), M.Sc. (Hort.). Thesis, Agricultural College and Research Institute, Madurai.

Samadia, D.K. 2011. Genetic variability studies in ridge gourd under arid environment. Indian J. Hort., 68 (2): 275-277.

Shivananada, M. M., M.B. Madalageri, S.S. Chikkur, A.b. Mohankumar and Yathiraj, K. 2013. Correlation and path co-efficient studies in pumpkin (Cucurbita moschata Dutch. Ex. Poir.). Int. J. Agric. Sci., 9 (1): 76-79.

Singh, D.K and Rajeshkumar. 2002. Studies on genetic variability in bottle gourd. Prog. Hort., 34 (1): 99-101.

Sirohi, P.S. and Ghorui, S. 1993. Gene effects of certain quantitative characters in pumpkin. Veg. Sci., 20 (2): 158-162.

Yadav, Y.C., S. Kumar, A. Kumar, R. Singh and Singh, R. 2010. Path coefficient studies and character association in bottle gourd. Ann. of Hort., 3 (1): 84-88.

\section{How to cite this article:}

Sampath, S. and Krishnamoorthy, V. 2017. Genetic Variability, Correlation and Path Analysis in Pumpkin (Cucurbita moschata Duch. ex. Poir). Int.J.Curr.Microbiol.App.Sci. 6(6): 30273035. doi: https://doi.org/10.20546/ijcmas.2017.606.360 